\title{
Use of the Hospital Anxiety and Depression Scale and the Taiwanese Depression Questionnaire for screening depression in head and neck cancer patients in Taiwan
}

\author{
This article was published in the following Dove Press journal: \\ Neuropsychiatric Disease and Treatment \\ 13 October 2016 \\ Number of times this article has been viewed
}

Yu Lee'

Yi-Shan Wu'

Chih-Yen Chien²

Fu-Min Fang ${ }^{3}$

Chi-Fa Hung'

'Department of Psychiatry, ${ }^{2}$ Department of Otolaryngology, ${ }^{3}$ Department of Radiation Oncology, Kaohsiung Chang Gung Memorial Hospital and Chang Gung University College of Medicine, Kaohsiung, Taiwan, Republic of China
Correspondence: Chi-Fa Hung Department of Psychiatry, Kaohsiung Chang Gung Memorial Hospital, I 23 Ta-Pi Road, Niao-Sung District, Kaohsiung City 833, Taiwan, Republic of China

$\mathrm{Tel}+88677317123$ ext 6316

Fax+88677326817

Email chifa@cgmh.org.tw
Objective: The purposes of this study are 1) to estimate the prevalence of common mental disorders including depressive disorder in patients with head and neck cancer (HNC) at baseline and at the 6-month follow-up and 2) to test the validity of two self-reported questionnaires, the Hospital Anxiety and Depression Scale (HADS) and the Taiwanese Depression Questionnaire (TDQ), for screening depression in patients with HNC.

Methods: Participants were recruited from the outpatient collaborative care clinic for HNC of a tertiary hospital in Taiwan between January 2010 and January 2011. Ninety-three patients with HNC were enrolled and assessed using the HADS, TDQ, and Structured Clinical Interview for Diagnostic and Statistical Manual of Mental Disorders, fourth edition, Patient edition, at baseline and at the 6-month follow-up. Conventional validity indices of the HADS and TDQ were examined.

Results: Our results showed that the validity of the TDQ was satisfactory and comparable to that of both the HADS depression subscale and the HADS total scale. The cutoff scores of the HADS and TDQ for screening possible depressive disorders were 8 and 15 , respectively. The areas under the receiver operating characteristic curve of the HADS and TDQ were mean $0.975 \pm 0.015$ and $0.966 \pm 0.019$, respectively. Thirteen participants $(14 \%)$ were diagnosed with depressive disorders at the 6-month follow-up, compared with $8.5 \%$ at baseline.

Conclusion: Our results indicate that both the HADS and TDQ are valid instruments for screening depression in patients with HNC.

Keywords: head and neck cancer, Hospital Anxiety and Depression Scale, Taiwanese Depression Questionnaire, validity, prevalence

\section{Introduction}

Head and neck cancer (HNC) is the sixth most common cancer worldwide and was responsible for an estimated 300,000 deaths in 2008. ${ }^{1}$ In the United States, $~ 52,610$ incident cases of HNC were estimated to occur in $2012 .^{2}$ In Taiwan, oral cancer ranked as the fifth most common cause of cancer mortality in 2013 and was the fourth most common cause of cancer mortality among men. ${ }^{3}$ The overall 5 -year survival rate for patients with $\mathrm{HNC}$ is one of the lowest among different cancers, and this has not significantly changed during the last 2 decades. ${ }^{4}$

Survival and quality of life are the main treatment goals for cancer patients. Previous studies have shown that patients with $\mathrm{HNC}$ are often comorbid with depressive disorders. ${ }^{5}$ The reported prevalence of depressive symptoms in HNC patients varied 
from $9 \%$ to $52 \%$, and depressive disorders were diagnosed in $3.7 \%-47 \%$ of patients. ${ }^{5-8}$ Depression in cancer patients is associated with poor quality of life and shorter survival time. Therefore, early screening to identify depression and the provision of effective treatment are crucial for holistic care of patients with HNC.

To date, most studies have used self-reported depression questionnaires to assess depression in cancer patients, but only a few studies have assessed depression using structured standardized clinical interviews as diagnostic instruments. ${ }^{6}$ The main reasons for using self-administered depression rating scales in those studies might be that they are less timeconsuming and less expensive for identifying depression in patients with cancer compared with standardized structured interviews. However, self-reported questionnaires are used for assessing "depressive symptoms" not "depressive disorder", raising the concern of their validity for diagnosing depression.

The Hospital Anxiety and Depression Scale (HADS) was initially designed to assess the psychological distress of medically ill patients. ${ }^{9}$ Previous studies have shown that the HADS is useful for screening psychiatric morbidity in cancer patients. ${ }^{10,11}$ The Mandarin version of the HADS was first used to detect depression in chronic headache patients and was found to have satisfactory validity. ${ }^{12}$ This version of the HADS was later used for screening minor mental disorders in a cancer hospital in Taiwan and was reported to have fair sensitivity and specificity. ${ }^{13}$ The validation of the Mandarin version of the HADS for detecting depression in patients with HNC has never been assessed.

We developed a culture-sensitive depression screening questionnaire, the "Taiwanese Depression Questionnaire" (TDQ) ${ }^{14}$ The TDQ has shown its ability to screen depression in a community sample; it had a Cronbach's $\alpha$ coefficient (internal consistency of reliability) of 0.90 , and the area under the receiver operating characteristic curve (AUC) was 0.922 . The 18-item TDQ had a sensitivity of 0.89 and a specificity of 0.92 at a cutoff point of $19 .{ }^{14}$ These results suggested that the TDQ can be adapted for screening depression in the community. We also completed a study examining the validity of the TDQ for detecting depression in chronic pain patients, which resulted in a satisfactory validity index. At a cutoff point of 24 , it achieved a sensitivity of $76.7 \%$, specificity of $85.2 \%$, positive predictive value (PPV) of $93.3 \%$, negative predictive value (NPV) of $57.5 \%$, efficiency of $79 \%$, and AUC of $0.829 .{ }^{15}$ During the last decade, the TDQ has been widely used as a screening instrument for the detection of depression in Taiwan ${ }^{16-18}$ and in Turkey. ${ }^{19}$ The TDQ has been applied to assess depression at oncological services in recent years and had fair validity. ${ }^{20}$ However, the validation of the TDQ to evaluate depression in patients with HNC has never been studied. Whether the HADS or TDQ can effectively detect depression in HNC patients should be further explored.

In addition, numerous somatic symptoms of depression overlap with those of cancer and the side effects of cancer treatments; thus, accurate detection of depression in these patients is difficult. Some studies have questioned the validity of the self-rated depression questionnaires in examining depression in medical patients, especially the somatic items of these rating scales. ${ }^{21,22}$ One study comparing the validity of the Beck Depression Inventory and TDQ for detecting depression in chronic pain patients found that two factors, the somatization tendency of patients and the somatic components of the questionnaires, influenced the validity of these questionnaires. ${ }^{15}$ Whether the TDQ total score or its cognitive/affective component is valid for screening depression in cancer patients remains unknown.

The aims of this study included 1) estimating the prevalence of depressive disorders among patients with HNC at baseline and at the 6-month follow-up and 2) testing the validity of the TDQ and HADS in identifying depression among HNC patients. Furthermore, we investigated whether the somatic items of the TDQ influenced the validity of screening depression.

\section{Methods \\ Participants}

This study used a prospective design with consecutive sampling. Participants were recruited from the outpatient collaborative care clinic for $\mathrm{HNC}$ at a tertiary hospital from March 2010 to July 2011. This hospital has 2,754 beds with yearly services to 5,000 cancer patients in southern Taiwan. The inclusion criteria were as follows: 1) patients newly diagnosed with HNC and 2) patients with the ability to understand the study procedure and complete the questionnaires. Participants with dementia or delirium were excluded.

\section{Assessments}

\section{Hospital Anxiety and Depression Scale}

The HADS has been accepted as an effective screening instrument for anxiety and depression and has been extensively used in clinical settings and psycho-oncology research. The HADS is composed of two seven-item subscales, one specifically targeting anxiety (HADS-A) and the other focusing 
on depression (HADS-D). ${ }^{9}$ The items excluded somatic symptoms and therefore avoided symptom overlap between somatic illnesses and mood disorders. Since both the HADS total scale (HADS-T) and the HADS depression subscale (HADS-D) can be used to detect depression, the validity of the HADS-T and HADS-D was analyzed separately.

\section{Taiwanese Depression Questionnaire}

The TDQ is a culture-sensitive self-reported instrument for screening depression in Taiwan. ${ }^{14}$ This questionnaire is composed of 18 items related to mood, sleeping problems, appetite, energy, interest in normal activities, crying and feelings about the future, and so on. The participants were asked to indicate whether each item has been experienced and how frequently on a 4-point Likert scale (ranging from 0 to 3 ). The TDQ had satisfactory reliability and validity in a community study and among patients with chronic pain. ${ }^{14,15}$

\section{Structured Clinical Interview for DSM-IV, Clinician Version}

Psychiatric diagnoses were made using the Structured Clinical Interview for Diagnostic and Statistical Manual of Mental Disorders, fourth edition (DSM-IV), Clinician Version (SCID-CV), a structured diagnostic interview based on DSM-IV criteria. $^{23}$ The SCID was designed to be administered by a clinician or mental health professional who has relevant professional training. The diagnosis was supplemented by the $D S M-I V$ criteria for primary insomnia (which was not included in the SCID). The SCID interview by a trained psychiatrist is regarded as a gold standard of psychiatric diagnosis.

\section{Procedures}

Ethical approval was obtained from the human research ethics committee of Chang Gung Memorial Hospital. Study procedures were as follows: 1) newly diagnosed patients visiting our collaborative care clinic were invited consecutively to take part in this study. After explaining the study procedure and aims, those who agreed to sign the informed consent form were enrolled in the study; 2) psychiatric diagnoses were made by a senior psychiatrist (YL) using the SCID; 3) the HADS, TDQ, and clinical and demographic data were collected by a trained research assistant. Patients who were diagnosed with dementia or delirium were excluded. The earlier mentioned questionnaires and structured diagnostic interview were completed at baseline (pretreatment period), the 3-month follow-up, and the 6-month follow-up, respectively.

\section{Statistical analyses}

The differences in demographic data and clinical characteristics between participants with and without depressive disorders were tested by chi-square or student's $t$-test, where appropriate. Cronbach's $\alpha$ was applied to evaluate the internal consistency of the HADS and TDQ. The Cronbach's $\alpha$ values of the HADS-T, HADS-D, and TDQ were $0.88,0.75$, and 0.84 , respectively, suggesting that these questionnaires were acceptable and had good reliability in this study. The SCID interview results were used as the "gold standard" of psychiatric diagnosis against the TDQ or HADS. Sensitivity, specificity, PPV, NPV, and overall misclassification rate (OMR) were presented as indices used to demonstrate the validity of the HADS and TDQ. Receiver operating characteristic (ROC) curve analysis was performed to optimize the cutoff points for the HADS and TDQ. Patients were categorized as cases and noncases, based on the various cutoff scores, with a pair of sensitivity and specificity values for each cutoff score. Sensitivity was plotted against the false positive rate for every possible cutoff point, where the largest AUC was weighed to have the best validity. ${ }^{24} \mathrm{~A}$ method developed by Hanley and McNeil ${ }^{25}$ was utilized to assess whether there was a difference in the AUCs between the two questionnaires, to compare their validity. A critical ratio $z$ was defined as:

$$
z=\frac{A_{1}-A_{2}}{\sqrt{\mathrm{SE}_{1}^{2}+\mathrm{SE}_{2}^{2}-2 r \mathrm{SE}_{1} \mathrm{SE}_{2}}}
$$

where $A_{1}$ and $\mathrm{SE}_{1}$ were the observed area and the estimated standard error of the $\mathrm{AUC}$ of one questionnaire; $A_{2}$ and $\mathrm{SE}_{2}$ were the corresponding values of the other questionnaire; and $r$ was the estimated correlation between $A_{1}$ and $A_{2}$. All analyses were conducted using SPSS for Windows Version 16.0 (IBM, Armonk, New York). The significance level was set at $P<0.05$.

\section{Results Sample characteristics}

A total of 119 patients were referred for the study; eleven declined to participate and two did not fulfill the inclusion criteria (one had recurrent cancer and the other had Alzheimer's disease), resulting in 106 patients who completed baseline measures. The response rate was $89 \%$. Those who declined to take part in the study included four who had privacy considerations and seven who lacked the time to complete the assessment. At the 6-month follow-up, three of the participants were deceased and ten were lost 
contact, resulting in 93 patients who completed the 6-month assessment. There was no statistically significant difference in sociodemographic data between these 93 participants and the 13 who lost follow-up. The follow-up rate was $87.7 \%$.

Of the 93 patients who completed the 6-month assessment, $86 \%$ were male. The average age of the subjects was $52.7 \pm 9.5$ years, and $26.9 \%$ were currently unemployed. Approximately $74.2 \%$ of the participants were married, and the mean educational level was $9.9 \pm 3.6$ years. Sixty-five percentage of the patients had advanced (stages III and IV) disease. Approximately $63.4 \%$ and $41.9 \%$ of the participants had the habit of smoking cigarettes and drinking alcohol, respectively. There was no difference in demographic and clinical variables between the depressed and nondepressed participants (Table 1).

At the baseline assessment, $54.7 \%$ of participants were diagnosed with a psychiatric disorder. At the first visit, adjustment disorder (19.8\%) was the most prevalent psychiatric disorder, followed by alcohol use disorder (18.9\%), depressive disorders (8.5\%), anxiety disorders $(7.5 \%)$, and primary insomnia $(2.8 \%)$. However, at the 6-month follow-up, only $38.7 \%$ of the participants had a psychiatric disorder. The most prevalent psychiatric disorder at the 6-month follow-up was alcohol use disorder (20.4\%), followed by depressive disorders (14\%), primary insomnia (4.3\%), anxiety disorders (2.2\%), and adjustment disorder (1.1\%). Major depressive disorder (12.9\%) predominated among the depressive disorders, followed by depressive disorder not otherwise specified (1.1\%). The proportion of major depressive disorder was $4.7 \%$ at the index evaluation, increasing to $9.6 \%$ at the 3-month follow-up and $12.9 \%$ at the 6-month follow-up. Approximately one-fifth of patients had adjustment disorder at baseline and this dropped to $1 \%$ at the 6-month follow-up (Table 2).

Table I Demographic and clinical characteristics of the depressive and nondepressive patients with head and neck cancer at the 6-month follow-up

\begin{tabular}{|c|c|c|c|c|c|}
\hline Variables & $\begin{array}{l}\text { Depressives N (\%), } \\
\mathbf{N}=13\end{array}$ & $\begin{array}{l}\text { Nondepressives } \mathbf{N}(\%), \\
\mathbf{N}=\mathbf{8 0}\end{array}$ & $\begin{array}{l}\text { Total N (\%), } \\
\mathbf{N}=93\end{array}$ & $\chi^{2} / t$-test* & $P$-value \\
\hline \multicolumn{6}{|l|}{ Sex } \\
\hline Male & II (84.6) & $69(86.3)$ & $80(86.0)$ & 0.025 & 1.000 \\
\hline Female & $2(15.4)$ & II (I3.7) & $13(14.0)$ & & \\
\hline \multicolumn{6}{|l|}{ Age range, years } \\
\hline$<40$ & I (7.7) & $7(8.8)$ & $8(8.6)$ & 0.505 & 0.777 \\
\hline $40-60$ & $10(76.9)$ & $54(67.4)$ & $64(68.8)$ & & \\
\hline$\geq 60$ & $2(15.4)$ & $19(23.8)$ & $21(22.6)$ & & \\
\hline \multicolumn{6}{|l|}{ Education } \\
\hline Elementary school & $4(30.8)$ & $23(28.8)$ & $27(29.0)$ & 0.152 & 0.927 \\
\hline High school & $8(6 \mid .5)$ & $48(60.0)$ & $56(60.2)$ & & \\
\hline Over college & I (7.7) & $9(11.2)$ & $10(10.8)$ & & \\
\hline \multicolumn{6}{|l|}{ Marital status } \\
\hline Unmarried & $4(30.8)$ & $20(25.0)$ & $24(25.8)$ & 0.194 & 0.735 \\
\hline Married & $9(69.2)$ & $60(75.0)$ & $69(74.2)$ & & \\
\hline Education, years, mean \pm SD & $8.8 \pm 3.9$ & $10.1 \pm 3.6$ & $9.9 \pm 3.7$ & 1.207 & 0.231 \\
\hline Unemployment & $4(30.8)$ & $21(26.3)$ & $25(26.9)$ & 0.116 & 0.742 \\
\hline Comorbid diseases & $8(61.5)$ & $43(53.8)$ & $51(54.8)$ & 0.274 & 0.601 \\
\hline Hypnotics use & $2(I 5.4)$ & $4(6.3)$ & $6(6.5)$ & 1.998 & 0.196 \\
\hline Alcoholism & $6(46.2)$ & $32(40.0)$ & $38(41.9)$ & 0.175 & 0.675 \\
\hline Smoking & $10(76.9)$ & $49(61.3)$ & $59(63.4)$ & 1.184 & 0.361 \\
\hline Betel nut use & $4(30.8)$ & $23(28.8)$ & $27(29.0)$ & 0.022 & 1.000 \\
\hline Past history of depression & 0 & $2(2.5)$ & $2(2.2)$ & 0.332 & 1.000 \\
\hline Family history of depression & $\mathrm{I}(7.7)$ & $4(6.3)$ & $5(5.4)$ & 0.159 & 0.537 \\
\hline Time since cancer diagnosis, weeks, mean \pm SD & $1.8 \pm 1.1$ & $2.3 \pm 1.4$ & $2.2 \pm 1.4$ & 1.251 & 0.214 \\
\hline \multicolumn{6}{|l|}{ Treatment received } \\
\hline Operation & $7(53.8)$ & $43(53.8)$ & $50(53.8)$ & 0.000 & 1.000 \\
\hline Chemotherapy & $9(69.2)$ & $46(57.5)$ & $55(59.1)$ & 0.637 & 0.425 \\
\hline Radiotherapy & II (84.6) & $56(70.0)$ & $67(72.0)$ & 1.186 & 0.340 \\
\hline CCRT & $9(69.2)$ & $43(53.8)$ & $52(55.9)$ & 1.087 & 0.297 \\
\hline \multicolumn{6}{|l|}{ Stage } \\
\hline Early (stages I and II) & $2(15.4)$ & $31(38.8)$ & $33(35.5)$ & 2.667 & 0.127 \\
\hline Advanced (stages III and IV) & II (84.6) & $49(61.3)$ & $60(64.5)$ & & \\
\hline
\end{tabular}

Note: *Student's t-test.

Abbreviations: CCRT, concurrent chemoradiotherapy; SD, standard deviation. 
Table 2 Psychiatric diagnoses of patients with head and neck cancer at the first visit, the 3-month follow-up, and the 6-month follow-up

\begin{tabular}{|c|c|c|c|}
\hline \multirow[t]{3}{*}{ Category and diagnosis } & \multicolumn{3}{|l|}{ Visit } \\
\hline & First visit & 3-month follow-up & 6-month follow-up \\
\hline & $\overline{\text { Total }(\mathrm{N}=106)(\%)}$ & Total (N=94) (\%) & Total (N=93) (\%) \\
\hline Depressive disorders & $9(8.5)$ & $23(24.5)$ & $13(14.0)$ \\
\hline Major depressive disorder & $5(4.7)$ & $9(9.6)$ & $12(12.9)$ \\
\hline Depressive disorder NOS & $4(3.8)$ & $14(14.9)$ & $\mathrm{I}(\mathrm{I} . \mathrm{I})$ \\
\hline Dysthymia & 0 & 0 & 0 \\
\hline Adjustment disorder & $21(19.8)$ & $4(4.3)$ & $\mathrm{I}(\mathrm{I} . \mathrm{I})$ \\
\hline Anxiety disorders & $8(7.5)$ & $2(2.1)$ & $2(1.1)$ \\
\hline Anxiety disorder NOS & $6(5.7)$ & $2(2.1)$ & $2(1.1)$ \\
\hline Generalized anxiety disorder & I (0.9) & 0 & 0 \\
\hline Acute stress disorder & I (0.9) & 0 & 0 \\
\hline Alcohol abuse & $20(18.9)$ & $20(21.3)$ & $19(20.4)$ \\
\hline Primary insomnia & $3(2.8)$ & $4(4.3)$ & $4(4.3)$ \\
\hline No diagnosis & $48(45.3)$ & $51(54.2)$ & $57(6 I .3)$ \\
\hline
\end{tabular}

Abbreviation: NOS, not otherwise specified.

\section{Optimal HADS-T, HADS-D, and TDQ cutoff scores and conventional validity index}

To determine the optimal cutoff point to detect depression in HNC patients, we drew ROC curves based on various cutoff points for the TDQ (Figure 1). The more the curve arched toward the upper left corner, the better the validity of the test in differentiating depression from nondepression. We found that $93.4 \%$ of the subjects were accurately classified at a cutoff point of 15 . The AUC was $0.966 \pm 0.019$ (margin of error, Figure 1). Using the same method, the

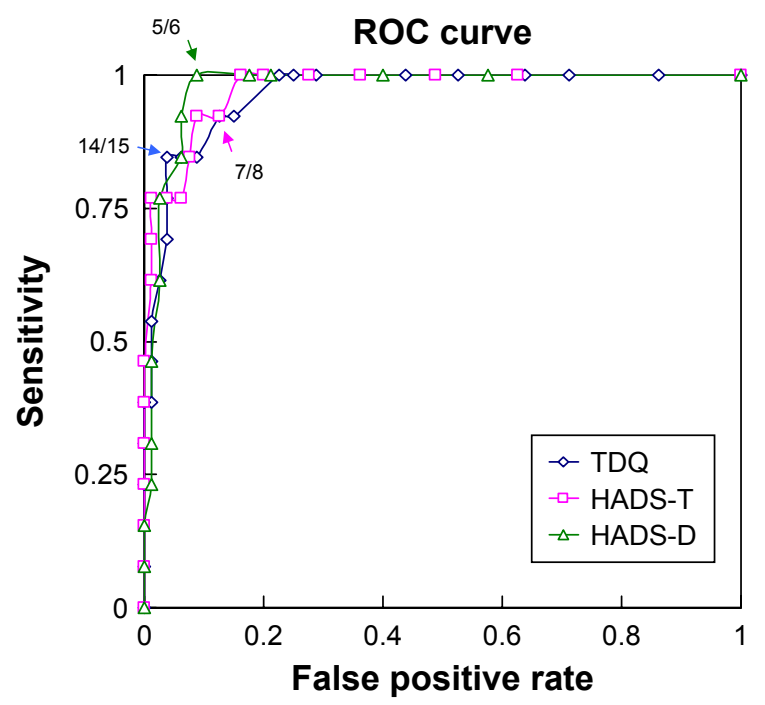

Figure I Receiver operating characteristic curve and optimal cutoff score for the TDQ, the HADS-T, and the HADS-D among patients with head and neck cancer. Abbreviations: TDQ, Taiwanese Depression Questionnaire; HADS, Hospital Anxiety and Depression Scale; HADS-D, HADS depressive subscale; HADS-T, HADS total scale; ROC, receiver operating characteristic. optimal cutoff points of the HADS-T and HAD-D were 8 and 6 , respectively. At these two points, $90.5 \%$ and $92.5 \%$ of the subjects were accurately classified. The AUCs of the HADS-T and HAD-D were $0.975 \pm 0.015$ and $0.976 \pm 0.014$, respectively (Figure 1).

We obtained the sensitivity, specificity, PPV, NPV, and OMR of the TDQ, HADS-T, and HADS-D using ROC curve analysis at various cutoff points. The results showed that a cutoff point of 15 for the TDQ achieved a sensitivity of $84.6 \%$, specificity of $96.2 \%$, PPV of $78.6 \%$, NPV of $96.3 \%$, and OMR of $6.4 \%$ (Table 3 ).

For the HADS-T, a cutoff point of 8 achieved a sensitivity of $92.3 \%$, specificity of $91.3 \%$, PPV of $63.2 \%$, NPV of $97.4 \%$, and OMR of $9.5 \%$. At the statistically obtained cutoff point of 6 for the HADS-D, sensitivity was $92.3 \%$, specificity 93.8\%, PPV 70.6\%, NPV 97.4\%, and OMR 7.5\% (Table 4). The data suggested that the validity of the TDQ, HADS-T, and HADS-D was good and satisfactory.

\section{Comparison of the validity of the TDQ, HADS-T, and HADS-D}

To test whether there was any difference in validity among the TDQ, HADS-T, and HADS-D, we did the following analyses using the method developed by Hanley and McNeil. ${ }^{25}$ Upon comparison of the AUCs of the TDQ, HADS-T, and HADS-D, the AUCs were 0.966 ( $\mathrm{SE}=0.018), 0.975$ (SE $=0.015)$, and $0.976(\mathrm{SE}=0.014)$ for the TDQ, HADS-T, and HADS-D, respectively. Our analysis showed that the AUC of the TDQ was very close to that of the HADS-D $(z=0.655$, $P=0.257$ ), indicating that the validity of the TDQ was comparable to that of the HADS-D in detecting depression in 
Table 3 Sensitivity, specificity, positive and negative predictive values, and overall misclassification rate of the TDQ total scale and TDQ cognitive scale

\begin{tabular}{|c|c|c|c|c|c|}
\hline Cutoff score & Sensitivity (\%) & Specificity (\%) & PPV (\%) & NPV (\%) & OMR (\%) \\
\hline \multicolumn{6}{|l|}{ TDQ total } \\
\hline II & 84.6 & 91.3 & 61.1 & 97.3 & 9.7 \\
\hline 13 & 84.6 & 93.8 & 68.8 & 97.4 & 7.5 \\
\hline 15 & 84.6 & 96.3 & 78.6 & 96.3 & 6.4 \\
\hline 16 & 76.9 & 96.3 & 76.9 & 95.1 & 7.5 \\
\hline 17 & 69.2 & 96.3 & 75.0 & 94.0 & 8.4 \\
\hline \multicolumn{6}{|l|}{ TDQ cognitive } \\
\hline 6 & 100.0 & 91.3 & 65.0 & 100.0 & 7.5 \\
\hline 8 & 92.3 & 93.8 & 70.6 & 96.2 & 8.3 \\
\hline 9 & 76.9 & 95.0 & 71.4 & 96.2 & 7.5 \\
\hline 11 & 76.9 & 96.3 & 76.9 & 96.3 & 6.4 \\
\hline
\end{tabular}

Abbreviations: TDQ, Taiwanese Depression Questionnaire; PPV, positive predictive value; NPV, negative predictive value; OMR, overall misclassification rate.

patients with HNC. We found that the AUCs of the TDQ and HADS-T were not significantly different $(z=0.551$, $P=0.291)$. This result suggested that the validity of the TDQ and HADS-T was comparable. Also, the AUC of the HADS-T was very close to that of the HADS-D $(z=0.117, P=0.453)$, implying that the validity of the HADS-T was comparable to that of the HADS-D.

\section{Validity of the cognitive/affective subscales of the TDQ}

To examine if the somatic items of the TDQ confounded its ability to identify depression in $\mathrm{HNC}$ patients, we performed additional ROC curve analyses of the cognitive/affective subscales of the TDQ.

The TDQ was divided into two subscales: cognitive/ affective and somatic, as determined by factor analysis in our previous study. ${ }^{15}$ For the cognitive component of the TDQ, the optimal TDQ cutoff point was 8 , using ROC curve analysis. The AUC of the TDQ was $0.977 \pm 0.014$ (margin of error). Ninety-two percent of subjects were accurately classified at a cutoff point of 8 , which achieved a sensitivity of $92.3 \%$, specificity of $93.8 \%$, PPV of $70.6 \%$, NPV of $96.2 \%$, and OMR of $8.3 \%$ (Table 3). These data demonstrated that the validity of the cognitive/affective subscales of the TDQ was satisfactory.

\section{Comparison of the validity of the total scale and cognitive/affective subscale of the TDQ}

To examine whether removing the somatic items from the TDQ would affect its ability to identify depression, we compared the AUC of the TDQ with that of the cognitive/ affective subscale of the TDQ using Hanley and McNeil' ${ }^{25}$ method. We found no significant difference between the AUC of the TDQ and the AUC of its cognitive/affective subscale $(z=0.712, P=0.239)$. This result suggested that the somatic

Table 4 Sensitivity, specificity, positive and negative predictive values, and overall misclassification rate of the HADS total scale (HADS-T) and HADS depression subscale (HADS-D)

\begin{tabular}{llllll}
\hline Cutoff score & Sensitivity (\%) & Specificity (\%) & PPV (\%) & NPV (\%) & OMR (\%) \\
\hline HADS-T & & & & & \\
6 & 100.0 & 83.8 & 50.0 & 98.6 & 14.4 \\
7 & 92.3 & 87.5 & 54.6 & 98.7 & 11.5 \\
8 & 92.3 & 91.3 & 63.2 & 97.4 & 9.5 \\
9 & 84.6 & 92.5 & 64.7 & 97.4 & 8.6 \\
II & 76.9 & 93.8 & 66.7 & & \\
HADS-D & & & & 100.0 & \\
4 & 100.0 & 82.5 & 48.2 & 98.7 & 14.0 \\
5 & 100.0 & 91.3 & 65.0 & 97.4 & 7.3 \\
6 & 92.3 & 93.8 & 70.6 & 96.3 & 8.3 \\
7 & 84.6 & 93.8 & 68.8 & 94.0 & 7.4 \\
\hline
\end{tabular}

Abbreviations: HADS, Hospital Anxiety and Depression Scale; PPV, positive predictive value; NPV, negative predictive value; OMR, overall misclassification rate. 
items did not significantly affect the validity of the TDQ in detecting depression in $\mathrm{HNC}$ patients.

\section{Discussion}

Our study suggested a high psychiatric morbidity among HNC patients from the initial pretreatment period to the posttreatment 6-month follow-up. The most prevalent psychiatric diagnosis of this study at baseline without cancer treatment was adjustment disorder (19.8\%), followed by alcohol use disorder (18.9\%) and depressive disorder (8.5\%). This is partially supported by Kugaya's report. ${ }^{26}$ Kugaya et $a^{26}$ investigated psychiatric disorders in 107 consecutive patients with $\mathrm{HNC}$ by structured psychiatric interview before the initial cancer treatment. They found that $13.1 \%$ of patients had adjustment disorder, $3.7 \%$ had major depression, and $33.6 \%$ met the criteria for alcohol dependence. ${ }^{26}$ The results of a meta-analysis of 66 studies conducted in oncological and hematological settings with 9,574 individuals across 14 countries were also in line with our results. ${ }^{27}$ Mitchell et $\mathrm{al}^{27}$ reported that a pooled prevalence of adjustment disorder and depression using DSM-IV criteria or International Classification of Diseases 10 was $19.4 \%$ and $16.3 \%$, respectively. The small discrepancies between previous studies and ours may be attributed to differences in study populations, diagnostic criteria, cancer types and stages, or research instruments.

The morbidity of depressive disorders changed during the 6-month follow-up: the prevalence of depressive disorders in our study was $8.1 \%$ at the first visit, increasing to $24.5 \%$ at the 3 -month follow-up, and dropping to $14 \%$ at the 6-month follow-up. Consistent with a previous review study, depression rates were particularly high at diagnosis, during treatment and even 6 years after treatment. ${ }^{5}$ The clinical implication for this finding is that cancer patients may suffer from depressive disorder at any disease stage, especially at 3 months posttreatment. Therefore, clinicians should pay more attention to those who are susceptible to depression and render a prompt diagnosis and treatment to improve their quality of life and even prolong survival.

The prevalence of depressive disorders in our study was $14.0 \%$, which is in line with that of previous hospital-based studies using standardized psychiatric interviews. To date, only five studies have used structured clinical interviews to estimate the prevalence of depressive disorder in patients with $\mathrm{HNC}$; the prevalence in those studies ranged from $3.7 \%$ to $43 \% .{ }^{8,26,28-30}$ Excluding one study that focused only on geriatric cancer patients, ${ }^{30}$ the prevalence in the other four studies was $3.7 \%, 20 \%, 26.1 \%$, and $43 \%$, respectively. Our result is similar to the results of these four studies, implying that the prevalence of depressive disorder in HNC patients obtained by the structured clinical interview is lower than the prevalence in studies using self-rated depression scales, which ranges from $9 \%$ to $52 \% .{ }^{5,6}$ Compared with those using structured clinical interviews, the strength of our study is that we recruited newly diagnosed $\mathrm{HNC}$ patients prospectively and consecutively, resulting in better generalization for clinical practice.

Our study demonstrated the efficacy of the Mandarin version of the HADS and TDQ as screening instruments for HNC patients, in terms of their good validity and AUC. The earlier mentioned results indicated that the validity of the TDQ, HADS-T, and HADS-D was good and comparable when used to identify depression in patients with HNC. The clinical implication of the satisfactory validity of earlier mentioned two self-reported questionnaires is the TDQ, a culture relevant instrument, as well as the HADS to detect depression in patients with $\mathrm{HNC}$ at Chinese oncological settings.

Previous Western country-based studies reported cutoff scores for the HADS-T that varied from to 8 to $20,{ }^{8,31,32}$ and cutoff scores for the HADS-D ranging between 5 and $8^{11,33}$ for detecting depression in cancer patients. Our results showed cutoff scores for the HADS-T and HADS-D of 8 and 6, respectively, which put the HADS-D within the range of prior reports, but the HADS-T at the lower limit of previous results. The possible explanation for our lower cutoff scores for the HADS-T is underreporting of depressive symptoms, possibly because the cancer patients were hesitant to admit depression so as to maintain a fighting spirit. ${ }^{34}$

A recent cancer study on the validity of the HADS in Taiwan reported that cutoff points of 8 and 15 were optimal for the HADS-D and HADS-T, with sensitivities and specificities of $72 \%$ and $86 \%$ for the HADS-D and $84 \%$ and $68 \%$ for the HADS-T, respectively. ${ }^{13}$ The cutoff scores in our study for both the HADS-D and HADS-T were lower than those in the earlier mentioned study. Possible explanations for this difference between the two studies are 1) the prior study focused on screening for psychological distress, but ours targeted depression, and 2) differences in subpopulations (cancer type and geographic regions) may have led to a higher prevalence of psychiatric morbidity in that study. ${ }^{13}$ Nevertheless, the sensitivity and specificity of the HADS in these two studies were from $68 \%$ to $92 \%$, which should be considered as suitable for identifying depression or psychological distress in cancer patients in Taiwan.

In a meta-analysis of the identification of depression in cancer patients, the HADS-T and HADS-D had a pooled 
sensitivity and specificity of $82.0 \%$ and $77.0 \% ; 71.6 \%$ and $82.6 \%$, respectively. ${ }^{10,31}$ In our results, the sensitivity and specificity of HADS-T and HADS-D were $92.3 \%$ and $91.3 \%$ and $92.3 \%$ and $93.8 \%$, respectively. Our findings, along with those of previous studies, suggested that HADS is a fairly good screening tool for depression in cancer patients, no matter whether total score or depression subscore.

The TDQ effectively screened depression in patients with $\mathrm{HNC}$ and showed satisfactory sensitivity, specificity, PPV, NPV, and AUC (0.966). This result is as good as that in our previous community-based study testing the validity of the TDQ (AUC $=0.92)^{14}$ and is probably better than that in a previous validity study on detecting depression in chronic pain patients $(\mathrm{AUC}=0.829) .{ }^{15}$ The validity indices are excellent, except the relatively low PPV. One should note that the PPV of a screening rating scale is dependent on the prevalence of the condition of interest, ie, PPV declines as prevalence decreases ${ }^{35}$ In our study, the prevalence of depressive disorders was $<20 \%$, which can account for the low PPV.

A previous study used the TDQ for screening depression in cancer inpatients and found that the AUC, sensitivity, specificity, PPV, NPV, and cutoff scores of the TDQ were $0.72,71.9 \%, 56.4 \%, 32.9 \%, 90.4 \%$, and 26 , respectively. ${ }^{20}$ Possible explanations for differences in the previous studies and our study are: 1) the previous study used the TDQ to screen for major depression and our study screened for all depressive disorders (including major depression, dysthymia, and depressive disorder NOS), which resulted in higher cutting scores in the previous study than in ours; 2) screening in the previous study was performed by several trained nursing staff members as a routine survey, and our study was administered by one trained research assistant, thereby avoiding inter-rater variations. This is a possible reason for our better validity index; and 3) the previous study included all cancer patients and ours enrolled only HNC patients.

The optimal, if not the best method for assessing depression in the medically ill, as well as in cancer patients, is still a subject of debate. Cancer patients frequently have somatic symptoms, including fatigue, insomnia, and poor appetite, which might influence the validity of self-administered depression questionnaires. Since the somatic items in the TDQ might confound the ability of these rating scales to detect depression in HNC patients, we examined the validity of the cognitive/affective component of this rating scale alone. After removing the somatic items, the validity index for the TDQ increased slightly, but not significantly, ie, the validity of the TDQ was not affected after removing the somatic items. Thus, the TDQ had good validity in its cognitive/affective component. A case-control study detected cognitive deficits in drug-free patients with major depressive disorder ${ }^{36}$ using the TDQ and found that the cognitive subdomain of the TDQ was correlated with cognitive function. The aforementioned study supports the satisfactory validity of the cognitive aspect of the TDQ, suggesting that it may accurately assess depression in cancer patients. The increasing bodies of research have found that depression in cancer patients is related to several biological factors although the findings are mixed and inconsistent. Such biological correlates include chemotherapeutic agents, natural killer cells, gamma interferon- $\gamma$, cytokines (such as interleukin 2 [IL-2], IL-6), omega 3 fatty acid, and so on. ${ }^{6,37,38}$ Besides detecting possible depression in patients with $\mathrm{HNC}$, we should further focus on relevant biological factors for understanding pathophysiology, predicting prognosis, and seeking more effective treatment in the future.

The high response rate and the use of a structured clinical interview by a psychiatrist are the strengths of the present study. However, certain limitations should be noted when interpreting these data. First, our participants were recruited from a tertiary hospital, so our results may not be generalizable to other treatment settings. Second, the sample size in this study was relatively small. Large-scale studies should be used in the future to assess and confirm our results.

\section{Conclusion}

Our findings indicate that the HADS and TDQ have good validity for identifying depression among HNC patients. Further studies of other cancer types are required to confirm our findings.

\section{Acknowledgments}

This study was supported by a grant from Kaohsiung Chang Gung Memorial Hospital, Taiwan (CMRPG891321). We also acknowledge the excellent performance of our research assistant, PY Chao.

\section{Disclosure}

The authors report no conflicts of interest in this work.

\section{References}

1. Ferlay J, Shin HR, Bray F, Forman D, Mathers C, Parkin DM. Estimates of worldwide burden of cancer in 2008: GLOBOCAN 2008. Int J Cancer. 2010;127(12):2893-2917.

2. Tarver T. Cancer Facts \& Figures 2012. American Cancer Society (ACS) $J$ Consum Health Internet. 2012;16(3):366-367.

3. Health Promotion Administration. Taiwan cancer registry [Online]; 2013.

4. Chen YJ, Chang JT, Liao CT, et al. Head and neck cancer in the betel quid chewing area: recent advances in molecular carcinogenesis. Cancer Sci. 2008;99(8):1507-1514. 
5. Haisfield-Wolfe ME, McGuire DB, Soeken K, Geiger-Brown J, De Forge BR. Prevalence and correlates of depression among patients with head and neck cancer: a systematic review of implications for research. Oncol Nurs Forum. 2009;36(3):E107-E125.

6. Archer J, Hutchison I, Korszun A. Mood and malignancy: head and neck cancer and depression. J Oral Pathol Med. 2008;37(5):255-270.

7. Duffy SA, Ronis DL, Valenstein M, et al. Depressive symptoms, smoking, drinking, and quality of life among head and neck cancer patients. Psychosomatics. 2007;48(2):142-148.

8. Katz MR, Kopek N, Waldron J, Devins GM, Tomlinson G. Screening for depression in head and neck cancer. Psychooncology. 2004;13(4): 269-280.

9. Zigmond AS, Snaith RP. The hospital anxiety and depression scale. Acta Psychiatr Scand. 1983;67(6):361-370.

10. Mitchell AJ, Meader N, Symonds P. Diagnostic validity of the Hospital Anxiety and Depression Scale (HADS) in cancer and palliative settings: a meta-analysis. J Affect Disord. 2010;126(3):335-348.

11. Özalp E, Soygür H, Cankurtaran E, Turhan L, Akbiyik D, Geyik P. Psychiatric morbidity and its screening in Turkish women with breast cancer: a comparison between the HADS and SCID tests. Psychooncology. 2008;17(7):668-675.

12. Juang KD, Wang SJ, Lin CH, Fuh JL. Use of the hospital anxiety and depression scale as a screening tool for patients with headache Zhonghua Yi Xue Za Zhi (Taipei). 1999;62(11):749-755.

13. Wang GL, Hsu SH, Feng AC, et al. The HADS and the DT for screening psychosocial distress of cancer patients in Taiwan. Psychooncology. 2011;20(6):639-646.

14. Lee Y, Yang MJ, Lai TJ, Chiu NM, Chau TT. Development of the Taiwanese Depression Questionnaire. Chang Gung Med J.2000;23(11): 688-694.

15. Lee Y, Lin PY, Hsu ST, Cing-Chi Y, Yang LC, Wen JK. Comparing the use of the Taiwanese Depression Questionnaire and Beck Depression Inventory for screening depression in patients with chronic pain Chang Gung Med J. 2008;31(4):369-377.

16. Chen KC, Yang YK, Lee IH, Yeh TL, Lu RB, Chen PS. Sexual dysfunction and physicians' perception in medicated patients with major depression in Taiwan. Depress Anxiety. 2008;25(9):E56-E62.

17. Wang LJ, Chen CK, Hsu SC, Lee SY, Wang CS, Yeh WY. Active job, healthy job? Occupational stress and depression among hospital physicians in Taiwan. Ind Health. 2011;49(2):173-184.

18. Yen YC, Rebok GW, Yang MJ, Lung FW. A multilevel analysis of the influence of Apolipoprotein E genotypes on depressive symptoms in late-life moderated by the environment. Prog Neuropsychopharmacol Biol Psychiatry. 2008;32(2):479-486.

19. Kursat S, Colak HB, Toraman A, Ekmekci C, Tekce H, Alici T. The relationship between depression-malnutrition and echocardiographicblood pressure parameters in chronic hemodialysis patients. Int Urol Nephrol. 2008;40(3):793-799.

20. Tu CH, Hsu MC, Chi SC, Lin HY, Yen YC. Routine depression screening and diagnosing strategy for cancer inpatients. Psychooncology. 2014; 23(9):1057-1067.

21. Geisser ME, Roth RS, Robinson ME. Assessing depression among persons with chronic pain using the Center for Epidemiological StudiesDepression Scale and the Beck Depression Inventory: a comparative analysis. Clin J Pain. 1997;13(2):163-170.
22. Wesley AL, Gatchel RJ, Polatin PB, Kinney RK, Mayer TG. Differentiation between somatic and cognitive/affective components in commonly used measurements of depression in patients with chronic low-back pain: let's not mix apples and oranges. Spine (Phila Pa 1976). 1991; 16(6 suppl):S213-S215.

23. First MB, Spitzer RL, Gibbon M, Williams JB. User's Guide for the Structured Clinical Interview for DSM-IV Axis I Disorders SCID-I: Clinician Version. Arlington, VA: American Psychiatric Pub; 1997.

24. Hsiao JK, Bartko JJ, Potter WZ. Diagnosing diagnoses: receiver operating characteristic methods and psychiatry. Arch Gen Psychiatry. 1989;46(7):664-667.

25. Hanley JA, McNeil BJ. A method of comparing the areas under receiver operating characteristic curves derived from the same cases. Radiology. 1983;148(3):839-843

26. Kugaya A, Akechi T, Okuyama T, et al. Prevalence, predictive factors, and screening for psychologic distress in patients with newly diagnosed head and neck cancer. Cancer. 2000;88(12):2817-2823.

27. Mitchell AJ, Chan M, Bhatti H, et al. Prevalence of depression, anxiety, and adjustment disorder in oncological, haematological, and palliativecare settings: a meta-analysis of 94 interview-based studies. Lancet Oncol. 2011;12(2):160-174.

28. Gilbert J, Haman KL, Dietrich MS, Blakely RD, Shelton RC, Murphy BA. Depression in patients with head and neck cancer and a functional genetic polymorphism of the serotonin transporter gene. Head Neck. 2012;34(3):359-364.

29. McCaffrey JC, Weitzner M, Kamboukas D, Haselhuhn G, Lamonde L, Booth-Jones M. Alcoholism, depression, and abnormal cognition in head and neck cancer: a pilot study. Otolaryngol Head Neck Surg. 2007;136(1):92-97.

30. Morton RP, Davies AD, Baker J, Baker GA, Stell PM. Quality of life in treated head and neck cancer patients: a preliminary report. Clin Otolaryngol Allied Sci. 1984;9(3):181-185.

31. Bjelland I, Dahl AA, Haug TT, Neckelmann D. The validity of the Hospital Anxiety and Depression Scale: an updated literature review. J Psychosom Res. 2002;52(2):69-77.

32. Kugaya A, Akechi T, Okuyama T, Okamura H, Uchitomi Y. Screening for psychological distress in Japanese cancer patients. Jpn J Clin Oncol. 1998;28(5):333-338.

33. Love AW, Grabsch B, Clarke DM, Bloch S, Kissane DW. Screening for depression in women with metastatic breast cancer: a comparison of the Beck Depression Inventory Short Form and the Hospital Anxiety and Depression Scale. Aust N Z J Psychiatry. 2004;38(7):526-531.

34. Holland JC, Breitbart WS, Jacobsen PB, Lederberg MS, Loscalzo MJ, McCorkle R. Psycho-Oncology. Oxford: Oxford University Press; 2010.

35. Tsuang MT, Tohen M, Gep Z. Textbook in Psychiatric Epidemiology. New York, NY: Wiley; 1995:229-242.

36. Hueng TT, Lee IH, Guog YJ, et al. Is a patient-administered depression rating scale valid for detecting cognitive deficits in patients with major depressive disorder? Psychiatry Clin Neurosci. 2011;65(1):70-76.

37. Yang M, Moon C. Neurotoxicity of cancer chemotherapy. Neural Regen Res. 2013;8(17):1606-1614.

38. Savard J, Villa J, Ivers H, Simard S, Morin CM. Prevalence, natural course, and risk factors of insomnia comorbid with cancer over a 2-month period. J Clin Oncol. 2009;27(31):5233-5239.
Neuropsychiatric Disease and Treatment

\section{Publish your work in this journal}

Neuropsychiatric Disease and Treatment is an international, peerreviewed journal of clinical therapeutics and pharmacology focusing on concise rapid reporting of clinical or pre-clinical studies on a range of neuropsychiatric and neurological disorders. This journa is indexed on PubMed Central, the 'PsycINFO' database and CAS,

\section{Dovepress}

and is the official journal of The International Neuropsychiatric Association (INA). The manuscript management system is completely online and includes a very quick and fair peer-review system, which is all easy to use. Visit http://www.dovepress.com/testimonials.php to read real quotes from published authors. 